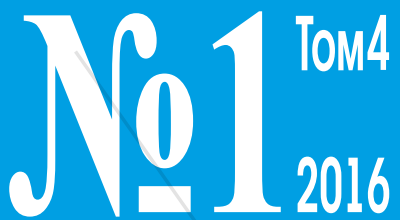

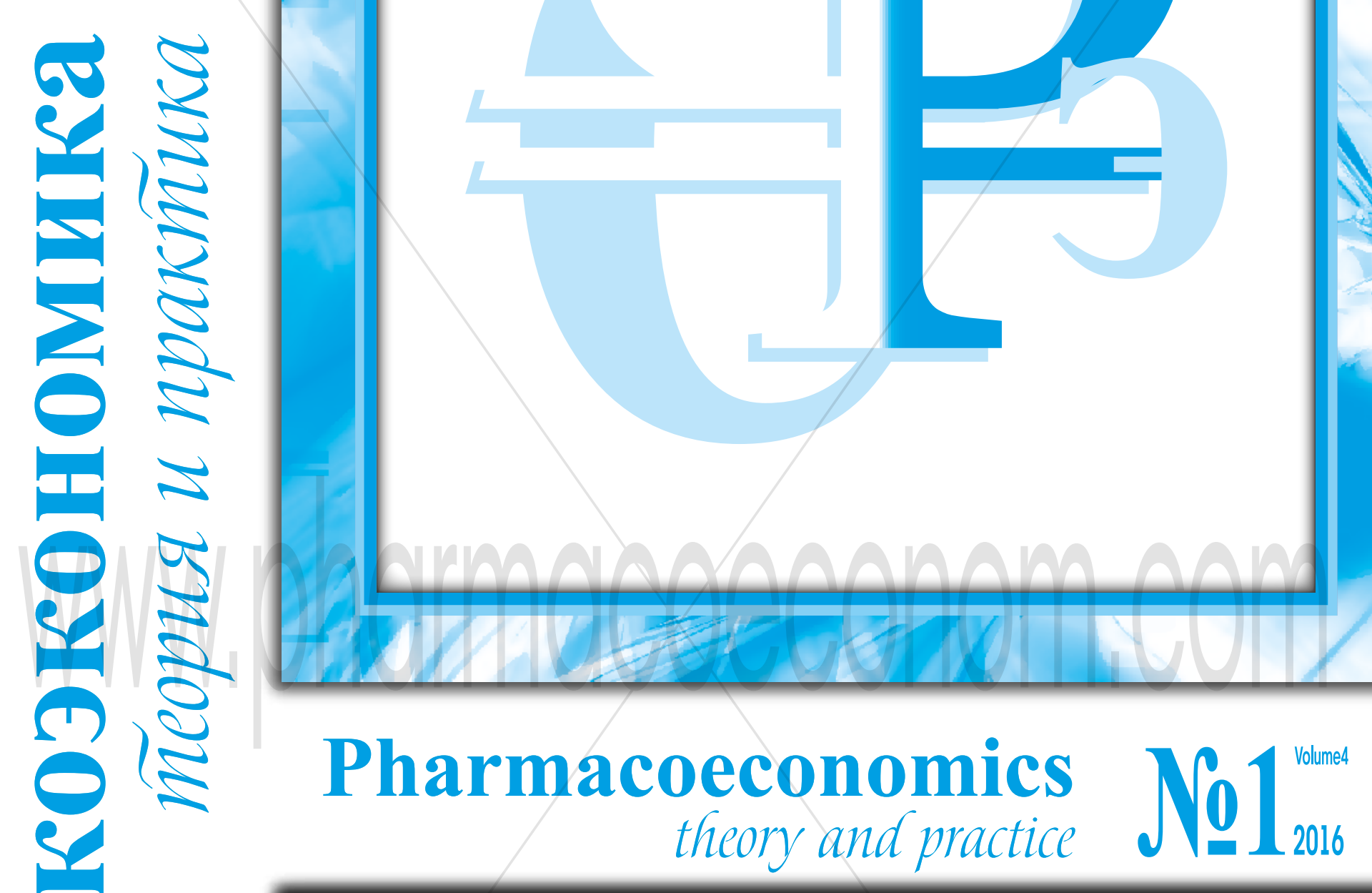

$\square$ МЕТОДОЛОГИЧЕСКИЕ ОСНОВЫ ФАРМАКОЭКОНОМИЧЕСКОГО МОДЕЛИРОВАНИЯ

$\square$ РЕЗУЛЬТАТЫ РОССИЙСКИХ ФАРМАКОЭКОНОМИЧЕСКИХ ИССЛЕДОВАНИЙ

口 МАТЕРИАЛЫ Х НАЦИОНАЛЬНОГО КОНГРЕССА С МЕЖДУНАРОДНЫМ УЧАСТИЕМ «РАЗВИТИЕ ФАРМАКОЭКОНОМИКИ И ФАРМАКОЭПИДЕМИОЛОГИИ В РОССИЙСКОЙ ФЕДЕРАЦИИ» 4-5 апреля 2016 г., г. Нижний Новгород 


\title{
METHODOLOGICAL ISSUES OF PHARMACOECONOMIC MODELING
}

\author{
Yagudina R.I, Serpik V.G. \\ I.M. Sechenov First Moscow State Medical University of the Ministry of Health of the Russian Federation
}

\begin{abstract}
:
The increase of practical application of pharmacoeconomics in the organization of medicinal provision the Russian Federation requires clarification of the definition of the concepts of pharmacoeconomics. Thus, in the context of the academic approach the term "pharmacoeconomics» can be defined as an independent science that studies in comparative terms the ratio between cost and effectiveness, safety and quality of life of patients with different treatment regimens or disease prevention. However, from the perspective of practical application it will be more accurate to consider pharmacoeconomics as a tool for decision support, providing decision-makers, relevant and reliable information in order to create from them a clear understanding of the situation and the possibility of taking appropriate optimal solutions. Herewith, wide opportunities of pharmacoeconomics are based on the application of pharmacoeconomic modeling. In this connection, the authors, based on their own practical experience create a domestic and adapted foreign foreign pharmacoeconomic models, set out the methodological basis of pharmacoeconomic modeling in this article in the form in which they meet the requirements and demands imposed by owners, using the results of pharmacoeconomic evaluations in the modern system of medicine provision in the Russian Federation
\end{abstract}

Key Words: modeling, pharmacoeconomic model, technical assignment, decision tree model, Markov model

Pharmacoeconomic modeling is a tool of pharmacoeconomic analysis, providing the best implementation of pharmacoeconomic evaluation in the practice of decision-making in health care.

The result of the use of pharmacoeconomic modeling is the development of an appropriate model. Therefore it is proposed to consider pharmacoeconomic modeling as the algorithm describing the process of developing a pharmacoeconomic model. The term "pharmacoeconomic model" is a comprehensive concept that includes a variety of semantic aspects. So, pharmacoeconomic model can be considered as the general definition of the term 'model' as a formalized simplified description of the object being studied. Also, the term model carries a prognostic nature of the obtain during data modeling. The model is called the mathematical apparatus that describes a particular object, often implemented through a variety of software products that provide interactivity model property, i.e. an opportunity in real time to make changes in the input data model and immediately get updated simulation results.

Nowadays the most relevant definition of pharmacoeconomic models is that it is interactive information computer program describing (and predicting) the consequences of the introduction in practical public health of a certain medicine. This definition of pharmacoeconomic model fits in the content of the relevant provision of the Decree of the Government of the Russian Feder- ation N871 dated 08.28.2014 When using mathematical modeling submitted in electronic form all the developed models and assumptions to develop appropriate models and calculation formulas used in the models."

As a practical tool of pharmacoeconomics, features and capabilities of pharmacoeconomic models are mainly determined, goals and objectives conducted during pharmacoeconomic studies, which are reflected when drafting the terms of reference. Thus, the starting point of the algorithm is creating pharmacoeconomic model and a prerequisite of its practical use is to write a proper technical specifications for pharmacoeconomic research tions:

In the technical assignment is recommended to reflect the following op-

- Title of the study

- The objects of the study - to list the studied medicines including reference medicines

- Model of patients - to specify the characteristics of patient groups for whom the drug is considered: disease/condition; separate form, if any; age and gender characteristics of patients, etc

- Research level - to indicate level of health care and the position in which pharmacoeconomic research is conducted. Pharmacoeconomic research can be conducted to the health system at the Federal level, regional and municipal levels, the level of health care institution. In the Russian Federation pharmacoeconomic study can take into account the position of the public health system, a separate program within the public health system, private insurance company or the patient.

- The functionality of pharmacoeconomic research is to specify what types of pharmacoeconomic analysis in addition to modeling should be used in the study and in what form the results of the pharmacoeconomic analysis should be submitted (e.g. is there a need for the automated generation of text report with the results of pharmacoeconomic analysis). Also features include predictive properties of the model - at what horizon it is planned to build a pharmacoeconomic model. This section of the technical specifications you can specify requirements to such specific properties of the model, as region specificity, the factor of penetration of new drug in the market, etc. In addition to the listed options, functionality reflect the requirements for the structure of pharmacoeconomic model, its software (by what software it needs to be implemented) and visual design. In terms of functionality it seems convenient to distinguish three groups of pharmacoeconomic models:

- a simple pharmacoeconomic calculators contain only the selected calculation methods of pharmacoeconomic analysis, without 
consideration of the characteristics of the patients (patient journey), regional characteristics. Pharmacoeconomic calculators do not have predictive properties and devoid of any graphical interface;

-analytical models of decision making (AMDM). The specified type of models has broad functionality. AMDM allows not only to carry out various types of pharmacoeconomic analysis, including the calculation of a variety of factors, from regional peculiarities of health systems to route the patient, etc., but also have a convenient visualization and other solutions that increase the degree of perception of the target audience presentherfindings. Another useful option of AMDM is significantincrease of the practicality of AMR as a tool for decision support is the ability of automatically generated text report containing pharmacoeconomic conclusions obtained on the basis of local data. As practical experience shows, AMDM most fully satisfy the needs of decision-makers, as once established, allows for pharmacoeconomic analysis taking into account peculiarities of each subject of the Russian Federation; - The main difference from the CORE models from AMDM is a more complex analysis of the effectiveness of the estimated drugs, taking into account many factors and it requires the involvement of a large number of clinicians and implies the inclusion of medical statisticians, etc. - CORE models, usually developed at the international level and are the most demanding and expensive in the development. For CORE models are inherent problems with adapting to the conditions of the Russian health system, because they are developed at the international level is a high probability of the use of indicators for which information is missing for Russia. In addition, for CORE models, the most typical problem is "black box", as in most cases, CORE model built on cloud type (calculations are performed on a remote server) and the computing model are not available to the user [1-6].

Formulated technical assignment, in fact, is a perfect vision of developed pharmacoeconomic model. However, it is necessary to take into account the fact that any model including pharmacoeconomic one is based on certain input data. In this connection, the ability to realize the pharmacoeconomic model in accordance with the original terms of reference determined by the availability of the required input data for modeling. If you are unable to receive these or other raw data technical assignment is adjusted.

Adjustment of technical specifications occurs as a result of information retrieval whose goal is to collect the basic data needed for model development

Note: the information search in the development of pharmacoeconomic model includes both the search and the choice of performance criteria for various types of pharmacoeconomic analysis ("cost-effectiveness" "costutility", "budget impact") and search for clinical, epidemiological and economic data used to reflect the route of the patient (see below) when building a pharmacoeconomic model.

The ongoing information retrieval specifies which raw data are available and which are not; It is also determined to obtain the required data indirectly by calculating them based on other parameters. In the absence of this possibility evaluated the acceptability of the use of the actual missing data modeling (eg, based in this case on the extrapolation of data or expert opinion) and its adequacy in connection with this assumption. If the required input data for pharmacoeconomic model described in the specification, can neither detect nor calculate or simulate, then resorted to adjusting the terms of reference in order to change the requirements for developed pharmacoeconomic model so that it functioned without initially specified initial data $[1,7]$.

After adjusting the technical assignment based on the results of information search they proceed to the description of the implementation of pharmacoeconomic model scenarios. Under the scenario pharmacoeconomic model is understood logically complete and mathematically independently described (by other calculations) path in the model of the input data to the output data.

Reflecting the effects of the implementation of a medicine in health care system, in fact, pharmacoeconomic model describes a certain section of the route (or all) of the patient associated with the use of the evaluated medicine. At the same time, the patient's route within the pharmacoeconomic model has two binding points - patient at entry point into the model and patient at exit point of the model (with the evaluation result output); if necessary the model can be reflected in additional intermediate stages between above described binding points. Taking into account that the patient depends on the characteristics of the route, one pharmacoeconomic model may have one scenario in this case to consider only one group of patients with fixed characteristics and several scenarios corresponding to different patient groups. For example, as for oncology pharmacoeconomic model may comprise one scenario, considering the patients receiving only the medicine in the first line therapy, and several scenarios, if the first line is analyzed in addition to the use in medicine of the second-line therapy.

Also, the division on the described scenarios in pharmacoeconomic models may depend on the stage of care, having separate scripts for patients receiving medical care at the outpatient stage or at the stage of hospital treatment. At the same time, both of these examples of pharmacoeconomic model may be implemented by a single scenario, and in this case, the model will reflect the integral treatment of patients in the first and second line therapy or treatment of patients in an outpatient or inpatient. The need for individual scenarios defined goals and objectives conducted pharmacoeconomic studies. If it seems appropriate discrete analysis of each (sub) group of patients in a pharmacoeconomic model implemented corresponding to each (sub) group of scripts. In the case where the interest is the assessment of a particular (sub) group of patients, or integral estimation provided that the features of the individual (sub) groups of patients do not affect the overall result in one scenario pharmacoeconomic model may be sufficient. Similarly, separation may require the individual scripts to different LP steam comparison between them.

The route of the patient most often described in pharmacoeconomic models (or tracked) using two modeling approaches: the decision tree model and Markov model (or its combinations). The major difference between these approaches is that the "decision tree" model examines the condition of patients, usually only at the input to the model and the output (the output in this case may be represented by a set of final states), so the time factor in the model is ignored (Figure 1). The final state in the "decision tree" model is the sum of a given sequence of developing events that occur with the patient during the movement along the route with a certain probability. The presence in the parameters of the model reflects the probability of its forward-looking (Figure 2).

Markov model, in turn, allows to analyze the patient's movement through-

\section{A. Decision tree model}

State in the input
State in the output

\section{B. Markov model}

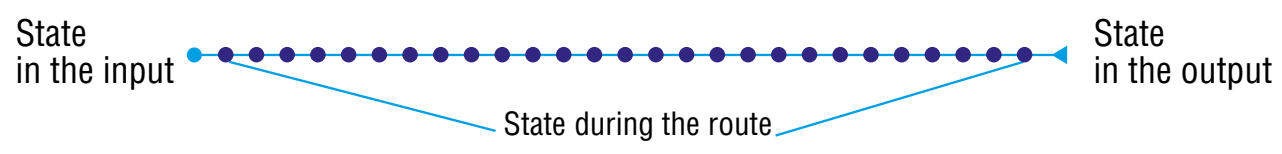

Figure 1. Fixing of patient route in decision tree model $(A)$ and Markov model (B) 


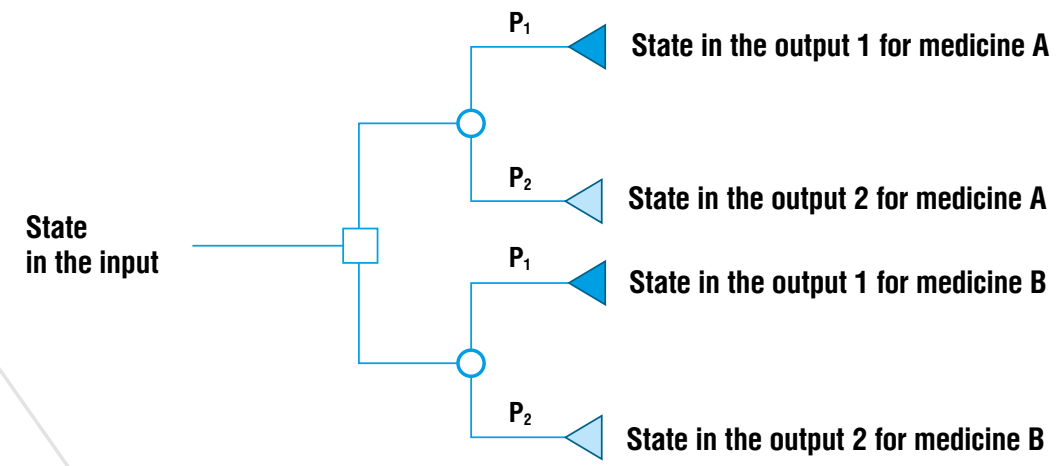

Keyword of the scheme of decision tree model

Sign of decision-making, reflecting the choice of any alternative treatment for patients

A sign of possible development of different events with the patient characterized by a certain value of the probability $\mathrm{p}$

The set of final states in the model

Figure 2. Scheme of decision tree model

out the route, thanks to the so-called Markov states (Figure 1). In the case of the pharmacoeconomic models Markov state is a fixed condition, in which may remain the patient (e.g., stage of disease, NB but not mandatory), whose characteristics are known. In this case, the condition of patients at the output of the model with pharmacoeconomics position is defined as the sum of the characteristics of Markov states in which the patients stayed for a time horizon considered in the model.

From this it follows that the Markov model is in contrast to the decision tree model takes into account the time factor. Also, there is another option of Markov models - Markov cycle. It determines how often in the Markov model is fixed stay of patients in a given Markov state. For example, within a given time horizon of pharmacoeconomic Markov model of one year, Markov cycle can be set to one or more days / weeks / months. Accordingly, the smaller the length of the Markov cycle, the greater the degree of detail achieved by the patient route. The third parameter in Markov model is the probability of Markov transition that characterizes the probability of transition from one patient in Markov state to another one within a Markov cycle (or probability of remaining in their current state).

In connection with Markov transitions, it is necessary to note one more feature of Markov states: some Markov state transitions are possible, while the other is not. For example, the transition from a state to a state of remission and exacerbation back possible, while the state after amputation of a lower limb of the patient makes it impossible to return to the previous state. Also Markov models emit adsorbing state (often the death of patients), which is defined as a Markov state from which possible transitions into any other state. Selecting both the quantity considered in pharmacoeconomic Markov model state, so Markov cycle duration is determined by the characteristics of the disease in question; values of Markov transitions in pharmacoeconomic models generally reflect the efficiency of studied drugs (Fig. 3).

Thus, pharmacoeconomic Markov model have higher accuracy in comparison with "decision tree" models, however, they are more difficult to accomplish and require more data. According to existing practice, use of "decision tree" models, is useful for pharmacoeconomic analysis of acute diseases, the duration of which is short (short time horizon of the model) that helps to minimize the factor of ignoring time in this type of models. Markov models should be used in pharmacoeconomic studies of chronic diseases, or diseases characterized by distinct stages, but the main condition for the implementation of Markov models is the availability of data on values of the Markov transitions. Possible combination of "decision tree" approach and the Markov modeling, when, for example, the entrance of patients into the model up to a certain intermediate stage is described by the "decision tree" model and then

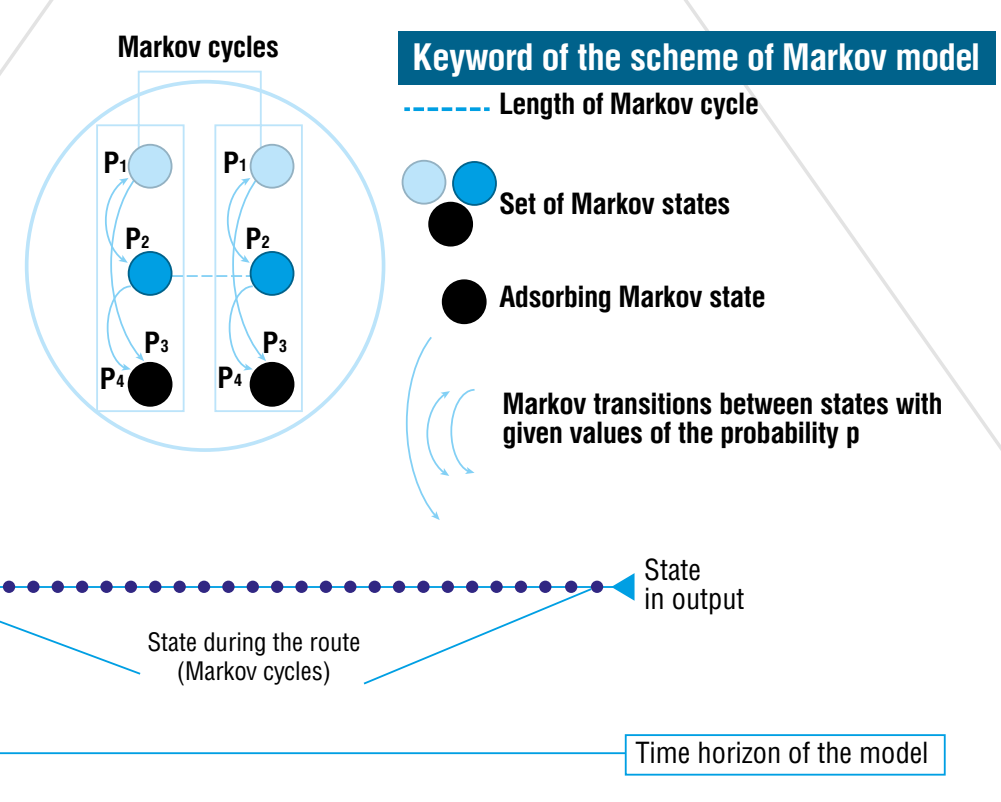

Figure 3. Scheme of Markov model 
pass into Markov model with all its characteristic attributes - Markov States, Markov cycles and transitions [5,7].

Possible scenarios include not only the route of the patient, but also incorporates the features of the pharmacoeconomic analysis. Implementation of models in pharmacoeconomic "cost-effectiveness" analysis will match one scenario, "budget impact" analysis is different. Moreover, the "cost-effectiveness" analysis based on various performance criteria within a pharmacoeconomic model, in fact, means creating different scenarios. A similar situation arises when accounting for costs in pharmacoeconomic models - one scenario may reflect the inclusion of only direct costs (in the case of valuation of medicines), whereas the other scenario is carried out from the position of the region within the same pharmacoeconomic models can include indirect costs. Provided for in pharmacoeconomic model of the use of different price level (registered maximum prices (wholesale/retail), auction prices) also involves creating a separate script for each price level (Fig. 4) [1-4,5]

The interactivity of pharmacoeconomic models is realized through their implementation through a software (SW). Thus, the opportunity to modify input data of the model in real time and to obtain pharmacoeconomic conclusions applicable to current local conditions, leading to high value of applied pharmacoeconomic models. There are different types of software for the implementation of pharmacoeconomic models, which can be divided into universal and specific. The first group includes various types of spreadsheets, such as MS Excel. An example of specialized software may serve as the program TreeAgePro. The main difference of specialized WS from universal ones is the presence of built-in units formula units of typology data and schematic rendering models for various types of pharmacoeconomic analysis. The universal advantage is that they provide flexibility in constructing models in the absence of templates and availability. As a rule, universal SW consists of the basic software of computers in a particular configuration by default, then as specialized SW must be installed additionally for a fee. Thus, in the case of specialized software, can be problems in demonstrating the pharmacoeco- nomic model was created on unprepared computers.

In this regard, in our view, for creating pharmacoeconomic models for future practical use and demonstration, the use of universal software is more preferable.

When creating pharmacoeconomic models an important property is not only interactivity, but also the usability of the developed model. Ease of use pharmacoeconomic model depends on its interface and structuring of the model. In particular, in an interactive pharmacoeconomic model is preferable to provide for an interaction model with the user. So, when you open the model useful to describe its properties (with particular attention to the assumptions and limitations in the simulation) and the sequence of work in it. Navigation within the model (moving between sections of the model) needs to be logical. In this case, it is conditionally possible to allocate two types of navigations in pharmacoeconomic models: sequential, in which the user when working with the model proposed the only plan of action that includes several consecutive stages (sections). When sequential navigation to move from this section of the model can only be on one forward or backward (Fig. 5).

Use of consistent navigation is convenient in pharmacoeconomic models containing the minimum number of scenarios. Unlike sequential navigation, free navigation in the model allows the user to choose models' section to work with (Fig. 6). Free navigation is preferred for pharmacoeconomic models, which is implemented in many scenarios.

Each section of the model should be clearly structured both by the type of information presented (introductory whether data (the input data on efficiency, input cost, introductory epidemiology, etc.) or conclusions (conclusions on the "cost-effectiveness" analysis, conclusions of "budget impact" analysis, etc.)) and its significance - you should arrange access to basic information, then to secondary. In addition to the optimization of the structure of pharmacoeconomic model, it should be noted the choice of the correct graphic design that will provide a better perception created pharmacoeconomic model. In order to improve

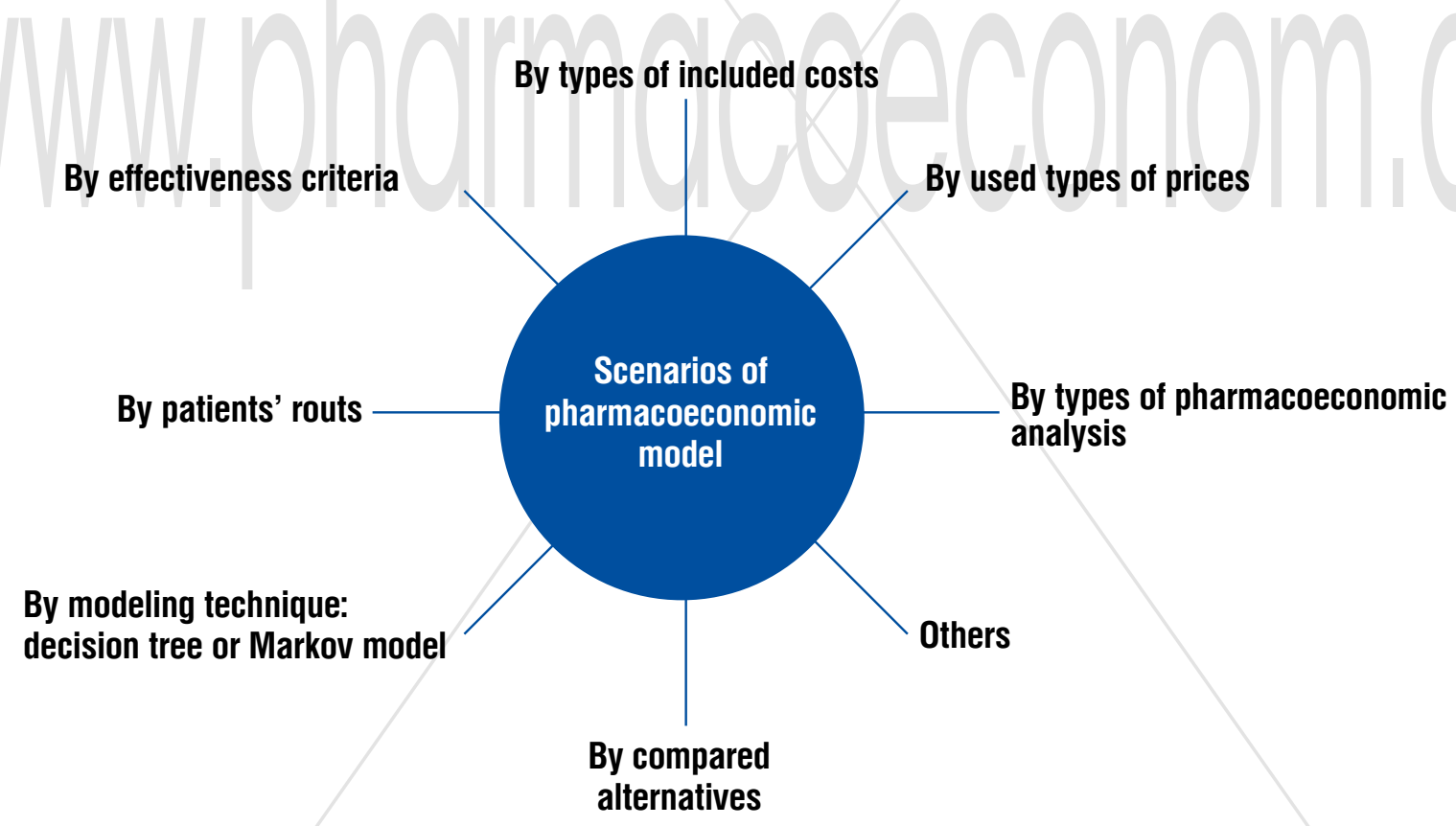

Figure 4. Classification of scenarios on pharmacoeconomic models

$\begin{gathered}\text { Sections, } \\ \text { unavailable for } \\ \text { transition }\end{gathered}$
$\begin{gathered}\text { Sections, } \\ \text { available for } \\ \text { transition }\end{gathered}$

Figure 5. Demonstration of consecutive navigation in pharmacoeconomic model 


\section{Sections,
unavailable for
transition}

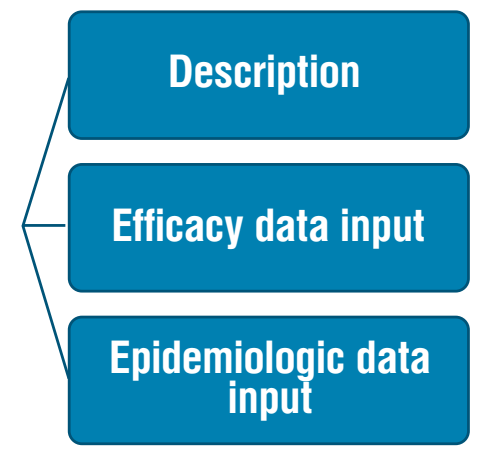

\section{Current section}

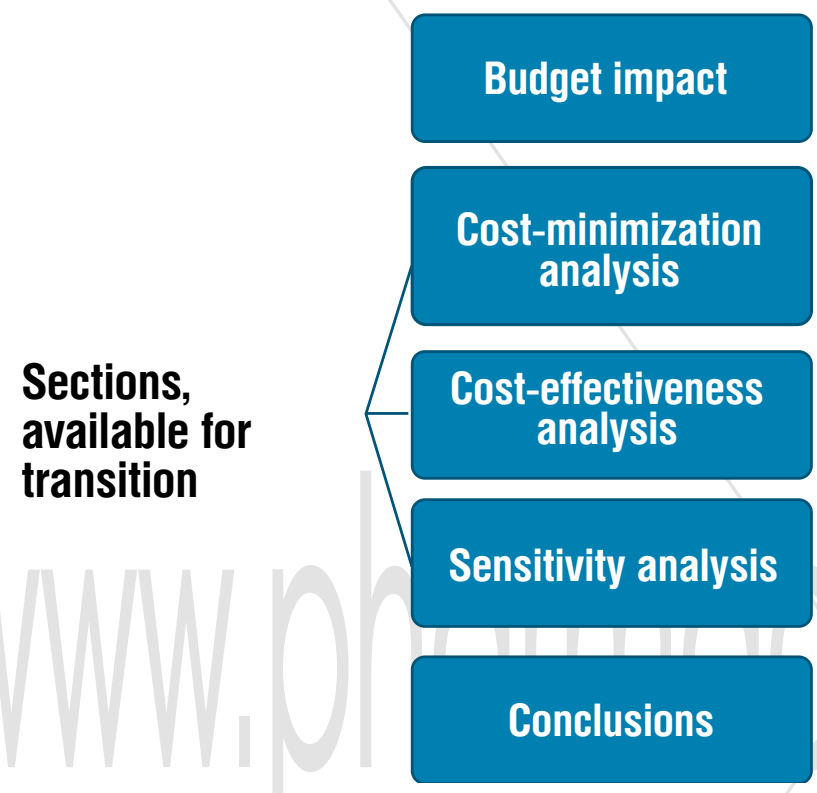

Figure 6. Illustration of freedom of navigation in pharmacoeconomic models the interface and design of pharmacoeconomic models they can be translated into flash technology, which adds dynamics and improves the visualization of the model. The possibility to automatically generate a text report with pharmacoeconomic conclusions made based on input of local data in pharmacoeconomic model, have also been achieved through programming [1-4].

Mathematically scenarios in pharmacoeconomic models are implemented using the device model, by which on the basis of input data pharmacoeconomic indicators are calculated. The volume and complexity of the mathematical apparatus is determined by the amount accounted in the model factors (input data), and scenarios implemented in the model methods of pharmacoeconomic analysis, the presence or absence of the procedure of discounting, etc. So in simple pharmacoeconomic calculators formula unit is reduced to the description of the calculations of "cost-effectiveness" analysis or "budget impact" analysis, whereas in the case of more sophisticated pharmacoeconomic models (AMDM, the CORE model) can be used by optimization algorithms and predictive calculations, including application of techniques of simulation. Specific formula-based machine requires the implementation of a mathematical approaches of modeling the type of "decision tree" model and Markov model. An interactive pharmacoeconomic model almost always involve the use of elements of Boolean algebra [7].

Also in the context of the mathematical description of pharmacoeconomic models we can distinguish deterministic and stochastic models. The first are characterized by the use of discrete data - given numerical values, whereas in stochastic models, calculations are made based on the given parameters of the distributions of certain variables (input data). The result of the deterministic models is point estimates, whereas stochastic models allow to perform for interval assessment in the form of ranges of possible values, each of which has its own probability of occurrence. Thus, stochastic models more effectively implement a predictive function, demonstrating all the possible outcomes in accordance with specified significant distributions of estimated parameters. In the context of creating pharmacoeconomic models means of software, the mathematical description of the model is a writing code of developed pharmacoeconomic model [7].

The results of the pharmacoeconomic modeling and the results of any pharmacoeconomic studies should be subjected to sensitivity analysis to determine their stability under possible change of input data. In this regard, it seems reasonable to develop in the framework of the established pharmacoeconomic model, the unit of the sensitivity analysis.

The final stage of development of pharmacoeconomic model is its validation for assessment of adequacy of reflection of the object described in the model and the resulting conclusions in relation to reality.

Thus, the correct result of the conducted pharmacoeconomic modeling, algorithm which is shown in figure 7 , is the creation of a pharmacoeconomic model is a powerful tool for decision support, significantly increasing the practical value of the pharmacoeconomic evaluation.

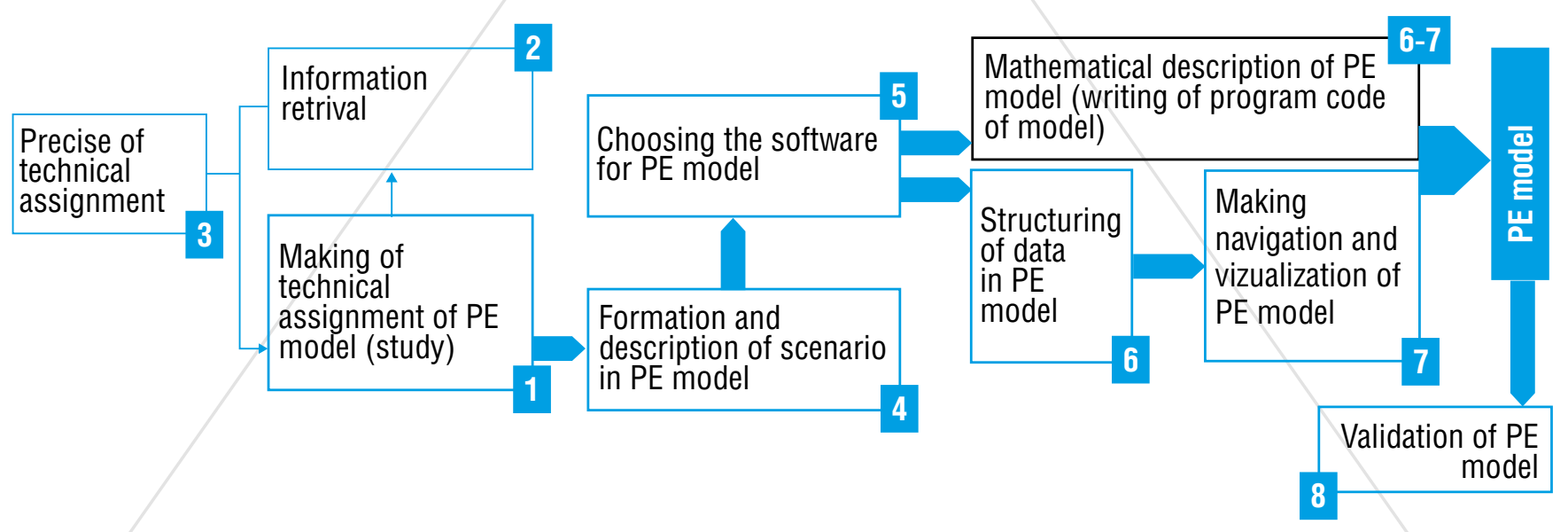

Figure 7. The algorithm for the development of pharmacoeconomic models 


\section{References:}

1. Yagudina R. I., Kulikov A. Yu., Arinina E. E. Pharmacoeconomics in Oncology. M.: Practica. - 2011. - 424 p.

2. Yagudina, R.I., Kulikov, A.Yu., Arinina, E.E. Pharmacoeconomics of the 2nd type diabetes. M.: "Medical Information Agency", 2011. - 352 pages

3. Yagudina, R.I., Kulikov, A.Yu., Serpik, V.G. Pharmacoeconomics in ophthalmology. M.: "Medical Information Agency", 2013. - 304 pages

4. Khabriev R.U., Kulikov A.Yu., Arinina E.E. Methodological basis of pharmacoeconomic analysis. Moscow: publisher "Medicine", 2011. - 128 pages.

5. Yagudina R.I., Serpik V.G. On the possibilities of combining budget impact analysis and cost-effectiveness analysis - development of «3D» pharmacoeconomic model // Pharmacoeconomics: theory and practice. - 2014. - Vol.2, №3. - P.9-13

6. Kulikov A.Yu., Litvinenko M.M. Methodological basics of modeling in the pharmacoeconomic studies: different levels of complexity and different value of the results obtained // Pharmacoeconomics: theory and practice. - 2014. - Vol.2, №2. - P.16-22

7. Hamdy A. Taha. Vvedenie $v$ issledovanie operatsiy [Operations Research An Introduction]. Moscow, Williams Publ., 2001, 912 p. (in Russ.) 